\title{
Multistep switching phase diagram of ferromagnetic ring structures
}

\author{
M. Kläui, ${ }^{\text {a) }}$ C. A. F. Vaz, and J. A. C. Bland ${ }^{\text {) }}$ \\ Cavendish Laboratory, University of Cambridge, Madingley Road, Cambridge CB3 OHE, United Kingdom \\ L. J. Heyderman and C. David \\ Laboratory for Micro- and Nanotechnology, Paul Scherrer Institut, CH-5232 Villigen PSI, Switzerland \\ E. H. C. P. Sinnecker and A. P. Guimarães \\ Centro Brasileiro de Pesquisas Fisicas, R. Xavier Sigaud 150, Rio de Janeiro 22290-180, Brazil
}

\begin{abstract}
We present a study of the phase diagram for the multistep switching occurring in micrometer-sized ferromagnetic ring structures. The transition from the double to the triple switching is experimentally determined as a function of the geometrical parameters. We fin that triple switching involving the vortexcore state is present only for a small range of geometrical parameters, since it requires a large fil thickness, a large ring width, and a small inner diameter. The double switching prevails for other geometries. The phase boundary between the switching regimes can be explained by the results of micromagnetic simulations.
\end{abstract}

\section{INTRODUCTION}

Mesoscopic magnetic elements have received considerable attention recently, in large measure due to the booming research activity in nanoscience and due to their potential for applications in nanotechnology. These applications include memory elements in high density storage media or miniaturized sensor elements, ${ }^{1,2}$ while from a more fundamental point of view, the small length scale and low dimensionality lead to enhanced physical properties over bulk materials. Intensive research has been devoted to understanding and controlling the magnetic properties of small ferromagnetic elements. A promising geometry is the ring shape and ring structures have recently been intensively investigated, since they exhibit a range of different magnetic states and switching processes. ${ }^{3-10}$ In addition to the flux-closur vortex state another state was found, termed the "onion" state, accessible reversibly from saturation and characterized by the presence of two opposite head to head walls. The detailed spin structure of these magnetization configuration has been directly imaged and different types of head to head domain walls have been found in the onion state. ${ }^{11,12}$ In addition to rings, which exhibit a double switching process between the vortex and the onion states, very wide and thick rings feature triple switching during which the vortexcore state is attained [a micromagnetic simulation of this state, where a complete vortex core is present in the ring is shown in Fig. 1 (top right)]. In this article we determine the phase diagram for the occurrence of the vortexcore state and hence the triple switching. The phase boundary between the different switch-

${ }^{a}$ Current address: Fachbereich Physik, Universitaet Konstanz, Universitaetsstrasse 10, D-78457 Konstanz, Germany; electronic mail: mathias@klaeui.de

${ }^{\text {b)} E l e c t r o n i c ~ m a i l: ~ j a c b 1 @ c a m . a c . u k ~}$ ing regimes (double and triple switching) are investigated and explained taking into account the switching processes as predicted by micromagnetic simulations.

\section{EXPERIMENT}

To fabricate arrays of ring structures, electron beam lithography is employed. In the firs step, a poly (methylmethacrylate) resist, spincoated on a naturally oxidized $\mathrm{Si}(001)$ substrate, is patterned using a Leica LION LV1 electron beam writer. To write the rings, the electron beam follows a circular single pixel path along the ring and the line width is determined by the electron beam dose and defocus. ${ }^{13}$ For pattern transfer, a liftoff process is used. Polycrystalline cobalt film with a $3 \mathrm{~nm}$ thick gold capping layer

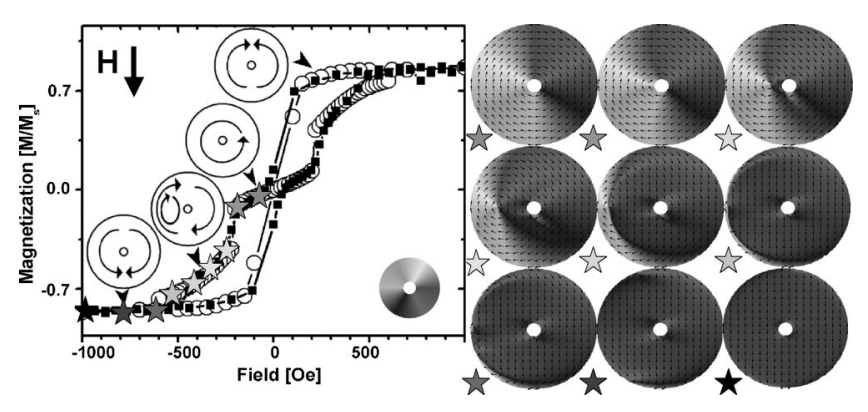

FIG. 1. (Left) $M-H$ hysteresis loop obtained by SQUID measurements showing triple switching in an array of very wide rings with a thick fil ( $D_{\text {ext }}=1.7 \mu \mathrm{m}, W=700 \mathrm{~nm}, t=32 \mathrm{~nm}$ polycrystalline Co; experimental data: black line, micromagnetic simulation: empty circles). SQUID measurements allow for direct magnetization measurements, corroborating earlier qualitative magneto-optical measurements (Ref. 12). The magnetization configuration are shown schematically (from top to bottom: onion, vortex, vortex core, and opposite onion state). (Right) The calculated magnetization configuration for the vortex to vortex core and the vortex core to reverse onion transitions are shown for the points indicated with the differently colored stars on the hysteresis loop (starting with the vortex state in the top left corner and then going left to right and top to bottom for states at increasingly negative field ). 


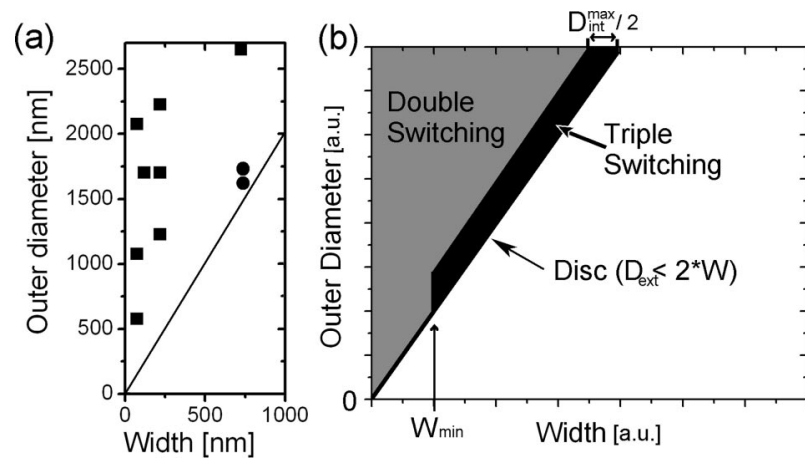

FIG. 2. (a) Experimental phase diagram of $32 \mathrm{~nm}$ thick polycrystalline Co rings. Most rings show a double switching (black squares) but wide rings with a small inner diameter $0<D_{\text {int }}<D_{\text {int }}^{\max }$ exhibit triple switching (black disks). The black line indicates the disk geometry and is the limit of the relevant part of the diagram (which is the top left part) and the line has in this outer diameter vs width diagram a slope with gradient 2 (on the line the outer diameter is $D_{\text {ext }}=2 \times W$ ). (b) Qualitative phase diagram in arbitrary units of the multistep switching region (thickness above the critical thickness where the transition from single to multistep switching occurs). The black line again corresponds to the disk geometry where $D_{\text {ext }}=2 \times W$. It can be seen that the triple switching (black area) only occurs in a limited region, where the ring is wide enough to accommodate a vortex core $\left(W>W_{\min }\right)$ and where the inner diameter is sufficientl small $\left(0<D_{\text {int }}<D_{\text {int }}^{\max }\right)$. As indicated in the diagram, the extent of the triple switching on the width axis is just $D_{\text {int }}^{\max } / 2$, since the right boundary is the transition to the disk geometry where the inner diameter is $D_{\text {int }}=0$ and the outer diameter is $D_{\text {ext }}=2 \times W$.

to prevent oxidation of the magnetic film were deposited on the patterned resist on silicon by molecular beam epitaxy in an ultrahigh vacuum deposition chamber (base pressure 3 $\times 10^{-10} \mathrm{mbar}$ ). The unwanted resist and magnetic material is then removed in acetone. Scanning electron microscopy images of rings fabricated in this way can be found in Ref. 14. The switching was then investigated by magneto-optical Kerr effect (MOKE) measurements on arrays of rings and superconducting quantum interference device (SQUID) measurements were carried out on some of the samples as well, confirmin the results of the MOKE measurements.

\section{RESULTS AND DISCUSSION}

Most rings investigated so far exhibit the standard double switching between the onion and vortex states. ${ }^{3-6}$ As shown in Ref. 14, very thin rings can exhibit a single switching, while in thick rings multistep switching (double or triple) occurs (the transition thickness being material and geometry dependent). In Refs. 11, 12 it was found that the triple switching occurs only in thick rings that are also very wide. Experimental and computational hysteresis loops of an array of such wide and thick rings (width $W=700 \mathrm{~nm}$, thickness $t=32 \mathrm{~nm}$ polycrystalline Co, and outer diameter $D_{\text {ext }}$ $=1.7 \mu \mathrm{m})$ are shown in Fig. 1. A clear triple switching is present and schematically the different magnetic states are displayed next to the hysteresis loops (onion state, vortex state, and vortexcore state). To quantify the occurrence of the triple switching, we have investigated systematically the type of multistep switching by varying the outer diameter and width of the rings while keeping the thickness constant. In Fig. 2(a) a multistep switching phase diagram is shown for $32 \mathrm{~nm}$ thick polycrystalline Co rings (the thickness being above the transition from single to multistep switching) with varying outer diameter and ring width (also note the relationship between width, outer and inner diameter: $D_{\text {ext }}=D_{\text {int }}$ $+2 \times W)$. As explained in Refs. 12, 11 the rings have to have a critical minimum width $W$ in order to accommodate for the vortex core and hence allow for triple switching. So, as expected, narrow rings exhibit double switching, while the triple switching only occurs in wide rings. With this in mind, it is however startling that in wide rings the triple switching is present for small outer diameters (e.g., $W=750 \mathrm{~nm}$ and $D_{\text {ext }}=1700 \mathrm{~nm}$ ) while the double switching prevails in rings with the same large width but with an increased outer diameter (e.g., $W=750 \mathrm{~nm}$ and $D_{\text {ext }}=2650 \mathrm{~nm}$ ). To understand this behavior, micromagnetic simulations of the actual switching processes have been computed using the OOMMF package $^{15}$ as detailed in Ref. 12 (the parameters used are saturation magnetization $M_{s}=1424 \times 10^{3} \mathrm{~A} / \mathrm{m}$, exchange constant $A=33 \times 10^{-12} \mathrm{~J} / \mathrm{m}$, and cubic cells of $4 * 4 * 4 \mathrm{~nm}^{3}$ ). The results of the simulations of the magnetization configu rations for the vortex to vortexcore and the vortexcore to onion transitions are shown in Fig. 1 (right). It can be seen that the vortex to vortexcore transition occurs by the nucleation of the vortex core at the inner edge. Then the vortex core is gradually pushed outwards as the applied fiel is increased, since the part of the ring where the magnetization is parallel to the applied fiel grows, until the vortex core is pushed out and annihilated, which corresponds to the vortex core to onion transition. To understand for which geometries the vortex to vortexcore transition occurs (which leads to a triple switching) and when the vortex to onion transition occurs (which leads to the double switching), we have to take into account the fact that the nucleation of the vortex core involves strong twisting of the spins at the inner edge to form the circular vortex structure. This is facilitated by a strong curvature of the inner edge, since the spins already form a curved structure and it is easier to twist them to form the complete vortex. Therefore a small inner diameter (strong curvature) favors the nucleation of a vortex core and hence the triple switching involving the vortex core state, as corroborated by our micromagnetic simulations of different inner diameter rings. Conceptually this then leads to the qualitative phase diagram (of rings with a thickness above the threshold where multistep switching occurs) shown in Fig. 2 (b): If the outer diameter $D_{\text {ext }}$ is equal to $2 \times W$ (width) then the element is a disk rather than a ring (black line); the relevant part of the diagram is the part above the line, which corresponds to rings. For small widths $W<W_{\min }$ the ring is too narrow to accommodate the vortex core and hence a double switching occurs ( $W_{\min }$ is to some extent thickness and material dependent). If the outer diameter is only slightly larger than $2 \times W$ then this corresponds to a small central hole and hence a high curvature of the inner edge and so triple switching occurs. If the outer diameter is much larger than $2 \times W$, then the central hole is large; in this case, there is only little curvature of the inner edge and hence in this case a double switching process occurs. From this it can be seen that in addition to the width $W$, the inner diameter plays a crucial role for the double to triple switching transition 
with triple switching occurring for rings with sufficientl small inner diameters $0<D_{\text {int }}<D_{\text {int }}^{\max }$ (but only in sufficientl wide rings).

\section{CONCLUSION}

In conclusion we have investigated the multistep switching phase diagram in micrometer sized Co rings. In agreement with earlier work we fin that triple switching involving the vortex core state is only present in sufficientl wide rings. What is more, it is not sufficien for the ring width to be wide enough to accommodate a vortex core in order for the ring to exhibit triple switching; another essential prerequisite is a small inner diameter. This is explained by the switching process, which involves the nucleation of a vortex core at the inner edge, which is facilitated by a small inner diameter. A qualitative phase diagram is derived, which elucidates that the triple switching can only occur in a limited range of geometrical parameters and for most geometries the double switching prevails.

\section{ACKNOWLEDGMENTS}

This work was supported by the EU ESPRIT program MASSDOTS, the EPSRC, the DFG (SFB 513) the PROFIX program (E.H.C.P.S.), the Gottlieb Daimler-and Karl BenzFoundation and the DAAD (M.K.).

${ }^{1}$ J.-G. Zhu et al., J. Appl. Phys. 87, 6668 (2000).

${ }^{2}$ M. M. Miller et al., Appl. Phys. Lett. 81, 2211 (2002).

${ }^{3}$ J. Rothman et al., Phys. Rev. Lett. 86, 1098 (2001).

${ }^{4}$ M. Kläui et al., Appl. Phys. Lett. 78, 3268 (2001).

${ }^{5}$ M. Kläui et al., Appl. Phys. Lett. 81, 108 (2002).

${ }^{6}$ S. P. Li et al., Phys. Rev. Lett. 86, 1102 (2001).

${ }^{7}$ S. Kasai et al., J. Magn. Magn. Mater. 239, 228 (2002).

${ }^{8}$ J. Bekaert et al., Appl. Phys. Lett. 81, 3413 (2002).

${ }^{9}$ X. Zhu et al., J. Appl. Phys. 93, 8540 (2003).

${ }^{10}$ M. Kläui et al., Phys. Rev. Lett. 90, 097202 (2003).

${ }^{11}$ M. Kläui et al., Phys. Rev. B 68, 134426 (2003).

${ }^{12}$ M. Kläui et al., J. Phys. D 15, 985 (2003).

${ }^{13}$ C. David et al., Microelectron. Eng. 46, 219 (1999).

${ }^{14}$ Y. G. Yoo et al., Appl. Phys. Lett. 82, 2470 (2003).

${ }^{15} \mathrm{The}$ public domain package is available at (gams.nist.gov/oommf) 